CONGER, A. D., AND FAIRGHILD, L. M. 1953. A quick freeze method for making smear slides permanent. Stain Tech., 28, 281-283.

HARDY, W. S., AND RUTHERFORD, T. 1962. Thermo-electric apparatus for the preparation of fresh-frozen tissue sections. Nature, 196, 785-786.

IOFFE, A. F. 1957. Semiconductor Thermo-elements and Thermo-electric Cooling. Infosearch Ltd., London.

RUTHERFORD, T., HARDY, W. S., AND ISHERWOOD, P. A. 1964. A thermo-electrically cooled microtome table and knife. Stain Tech., 39, 185-190.

\title{
MAINTENANCE OF HISTOCOMPATIBILITY POLYMORPHISMS
}

\author{
FREDERICK E. WARBURTON \\ Department of Biological Sciences, Columbia University, New York 27, N.Y.
}

Received 17.ix.67

\section{INTRODUGTION}

Billington (1964) and James (1965) have shown that the placenta of a mouse is larger if the foetus is antigenically incompatible with the mother (i.e. if the mother would reject skin grafted from an individual of the foetal genotype) than if it is compatible. Assuming this to confer a selective advantage on incompatible foetuses, Clarke and Kirby (1966) showed that a balanced polymorphism can be maintained at a histocompatibility locus with two alleles. I will show that a balanced polymorphism with an indefinitely large number of alleles can be maintained if it benefits a foetus to elicit an immunological reaction from its mother.

\section{ANALYsis}

Consider a locus with $n$ alleles, $a_{1} \ldots a_{i} \ldots a_{n}$, with population frequencies $p_{1} \ldots p_{i} \ldots p_{n}$. A foetus is incompatible with its mother if it possesses an allele not present in the mother. Let the relative fitnesses of compatible and incompatible foetuses be $1-s$ and 1 , respectively.

If a sperm carries the allele $a_{1}$, and the mother has the genotype $a_{i} a_{j}$, the zygote will be compatible if $i=1$ or $j=1$ or both. The probability that an $a_{1}$ sperm will enter into a compatible zygote is therefore $1-\left(1-p_{1}\right)^{2}$, or $2 p_{1}-p_{1}^{2}$.

If an egg carries the allele $a_{1}$, two cases must be considered:

1. The female is $a_{1} a_{1}$. The probability of this, given that the egg is $a_{1}$, is $p_{1}$. The zygote is compatible if the sperm carries $a_{1}$. The probability of both these events occurring is $p_{1}^{2}$.

2. The female is $a_{1} a_{i}(i \neq 1)$. The probability of this is $p_{i}$. The zygote is compatible if the sperm carries $a_{1}$ or $a_{i}$; the probability of this is $p_{1}+p_{i}$.

The net probability that an egg carrying $a_{1}$ will become a compatible zygote is the sum of these, with the probabilities in case 2 summed over all values of $i \neq 1$; i.e.

$$
p_{1}^{2}+\sum_{i \neq 1}^{n}\left(p_{1} p_{i}+p_{i}^{2}\right) \text {, or } p_{1}-p_{1}^{2}+\sum_{i=1}^{n} p_{i}^{2} .
$$

The mean relative fitness of the allele $a_{1}$, i.e. the weighted mean fitness of all zygotes into which $a_{1}$ enters, is the unweighted mean of the fitnesses of 
the zygotes into which $a_{1}$ is carried by a sperm and those into which it is carried by an egg:

$$
w_{1}=1-\frac{s}{2}\left[3 p_{1}-2 p_{1}^{2}+\sum_{i=1}^{n} p_{i}^{2}\right]
$$

The mean relative fitness of the population is:

$$
\bar{w}=1-s\left[2 \underset{i=1}{n} p_{i}^{2}-\sum_{i=1}^{n} p_{i}^{3}\right],
$$

which is a maximum if all alleles are equally frequent.

As any allele of less than average frequency has more than average fitness, and vice versa a balanced polymorphism can be maintained. At equilibrium, all alleles will be equally frequent.

If the population is at equilibrium with $n$ alleles, an additional allele $a_{x}$ introduced at very low initial frequency has a selective advantage,

$$
w_{x}-\bar{w}=s\left(3 / 2 n-1 / n^{2}\right),
$$

which is a decreasing function of $n$. Similarly, as the number of alleles in the system increases, the selection coefficients tending to restore them to their equilibrium frequencies after a disturbance decrease. If new histocompatibility alleles often arise by mutation, one would expect the number of alleles in the system to increase until the selection coefficients became too small to counteract random sampling effects. The system could then persist indefinitely, with its gene frequencies varying almost randomly, but with selection acting to incorporate new mutant alleles into the population whenever any were lost by chance. In this state, it would be hard to distinguish the system from a system of selectively neutral alleles.

\section{Summary}

1. A polymorphism involving many histocompatibility alleles could be maintained if the foetus benefits from an immunological reaction against the placenta by the mother.

2. Glearly, such an explanation can apply only to mammals and as histocompatibility polymorphisms are widespread in all vertebrate classes, some more widely applicable explanation remains to be found.

\section{REFERENGES}

BILLINGTON, w. D. 1964. Influence of immunological dissimilarity of mother and foetus on size of placenta in mice. Nature, 202, 317-318.

CLARKE, BRYAN, AND KIRBY, D. R. S. 1966. Maintenance of histocompatibility polymorphisms. Nature, 211, 999-1000.

JAMES, D. A. 1965. Effects of antigenic dissimilarity between mother and foetus on placental size in mice. Nature, 205, 613-614. 